\title{
Stroke without dissection from a neck holding manoeuvre in martial arts
}

\author{
M O McCarron, J Patterson, R Duncan
}

\begin{abstract}
Carotid artery trauma is a known cause of stroke in young people. The vessel may occlude, dissect or shower thrombotic emboli into intracranial vessels. This paper reports the use of single photon emission computed tomography (SPECT) imaging in a 29 year old man who developed an embolic stroke after neck holding manoeuvres at a martial arts class. Awareness of the potential consequences of these procedures is matched by the need for rapid and accurate diagnosis of stroke now that thrombolytic and neuroprotective treatments are emerging, which are effective only within a short time window.

(Br F Sports Med 1997;31:346-347)
\end{abstract}

Keywords: stroke; martial arts; single photon emission computed tomography

Neck injury from many diverse causes can interrupt carotid blood flow and disturb cerebral perfusion. Non-penetrating trauma can cause endothelial damage followed by thrombosis and embolisation. Strokes have been reported after attempted strangulation ${ }^{1}$ and automobile safety belt injuries. ${ }^{2}$ Martial arts have a high rate of head injury, ${ }^{3}$ but this report shows the potential serious outcome from an apparently trivial neck injury.

Stroke is a clinical diagnosis of a focal neurological deficit resulting from a vascular lesion and lasting longer than 24 hours. Different neuroimaging modalities such as computed tomography (CT) and single photon emission computed tomography (SPECT) can help secure the diagnosis. This combination of investigations can influence early treatment in suspected cases by excluding haemorrhage and confirming an ischaemic stroke respectively.

\section{Departments of \\ Neurology \\ M O McCarron \\ R Duncan}

and Clinical Physics

J Patterson

\section{Institute of}

Neurological Sciences,

Southern General

Hospital, Glasgow

G51 4TF

Correspondence to:

Dr R Duncan.

Accepted for publication 28 July 1997

\section{Case report}

A 29 year old man presented with a 16 hour history of left frontal headache, nausea, transient right visual field defect, and a non-fluent dysphasia. On the previous day he had attended a martial arts class where he participated in six neck holding manoeuvres, which he volunteered were stronger than usual. His opponent held his neck from behind in a brace-like fashion with a flexed left arm.

There was no family history of stroke. He did not smoke cigarettes, denied any illicit drug abuse, and consumed 12 units of alcohol per week. He was neither diabetic nor hypertensive and had no history of migraine or previous neurological deficit. Examination revealed an initial fever $\left(39^{\circ} \mathrm{C}\right)$, which resolved quickly, and a mild non-fluent dysphasia. There was no external neck injury or neck stiffness and no visual field defect was identified. Clinically, a stroke was suspected.

Compted tomography on admission was normal, confirming that no haemorrhagic lesion was present. At this stage aspirin was started at $150 \mathrm{mg} /$ day. SPECT of cerebral perfusion (after intravenous injection of $500 \mathrm{MBq}$ of technetium-99m HMPAO (Ceretec) and imaging with a Strichmann SME810 multidetector SPECT system) carried out the following day showed abnormally high perfusion in the left temporo-occipital regions (fig 1C) with a small area of reduced perfusion in the high left parietal region (fig 1D). A magnetic resonance (MRI) investigation performed four days later showed (fig 1A and 1B) several small areas of prolonged T2 signal within the left cortex, compatible with infarction in the distribution of the left middle cerebral artery following multiple cortical emboli. A cerebral angiogram excluded a dissection. Injection of contrast into the left internal carotid artery revealed an early arteriolar blush, corresponding to one of the MRI lesions and representing non-nutritional shunting of blood. A dominant left posterior communicating artery supplied the posterior cerebral artery. There was no evidence of stenosis or kinking of vessels. Follow up SPECT on day 60 showed the evolution of the ischaemic lesions (fig $1 \mathrm{E}$ and $1 \mathrm{~F}$ ), to a slight hypoperfusion in the lower sections and a well demarcated hypoperfused area in the parietal region consistent with the lesion seen on MRI (fig 1A). These appearances represent progression to infarction.

A search for stroke aetiology (erythrocyte sedimentation rate, echocardiography, protein C and S, antithrombin III, platelet count, coagulation screen, antiphospholipid antibody, and factor V Leiden) was unsuccessful. Two months after the event the patient had fully recovered.

\section{Discussion}

Immediate injuries from martial arts are usually easy to recognise. ${ }^{4}$ Non-penetrating carotid trauma can, however, present a diagnostic challenge because of the lack of external indications, a delay in the manifestations of clinical signs, and normal CT of the brain, ${ }^{2}$ all of which occurred in this patient. Carotid artery vulnerability has been demonstrated with prolonged lateral flexion of the head during a telephone call resulting in carotid artery dissection. ${ }^{5}$ Angiography excluded dissection in our patient. The history and imaging 


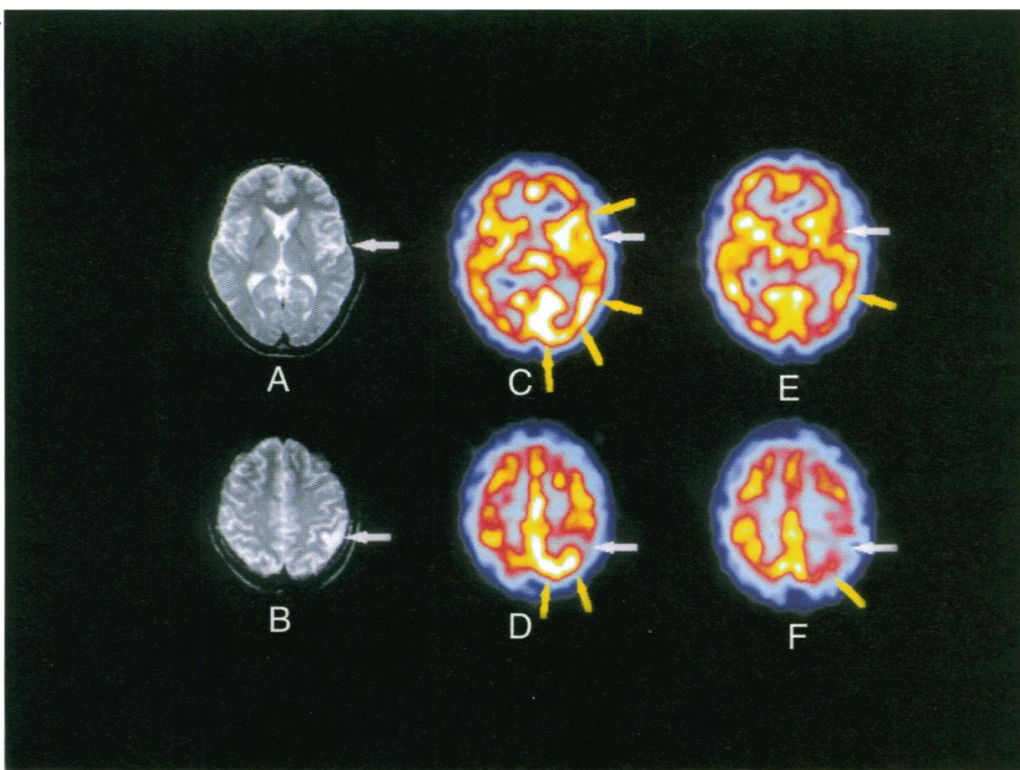

Figure 1 Magnetic resonance (MRI) and SPECT images at two axial levels in the brain - top row through the basal ganglia, bottom row through the parietal region. The $S P E C T$ images were taken on day 2 ( $C$ and $D)$ and day 60 ( $E$ and $F)$. MRI

demonstrates small areas of infarction (white arrows), which are also seen on the SPECT images. Areas of hyperperfusion seen on day 2 on the SPECT scan (yellow arrows) become slightly hypoperfused at day 60, confirming that damage has occurred.

findings strongly suggest, however, that the emboli arose from endothelial disruption and local thrombosis in the left carotid artery.

Despite its well defined role in the detection of haemorrhage the sensitivity of CT to acute ischaemic lesions is poor. CT usually does not show significant changes in parenchymal density until 24 hours after the event, although subtle changes can be seen in some large infarctions earlier than that. In some ischaemic events the CT images never become abnormal. In contrast, SPECT is able to demonstrate the primary perfusion defect from the moment it happens, provided it affects a large enough region $(>1 \mathrm{~cm})$ to be detected by this lower resolution technique. ${ }^{6}$ The appearance of the SPECT abnormality may change with time, as in our patient. After a period of ischaemia "luxury perfusion" may develop, ${ }^{7}$ where the perfusion may be much higher than the surrounding normal brain (fig $1 \mathrm{C}$ and 1D). This phenomenon is indicative of reperfused but damaged tissue. Later, the hyperperfusion subsides and may leave either a considerably hypoperfused area that correlates with the structural changes and relates to gross infarction or a slightly hypoperfused area that indicates only partial neuronal loss, at a level not readily seen by $M R I$.

Awareness of the potential consequences of neck injury is vital to encourage prevention and to highlight the need for rapid and accurate diagnosis as well as appropriate treatment. With the emergence of powerful new thrombolytic and possibly neuroprotective treatments the role of imaging will increase.

Thrombolysis with tissue plasminogen activator, is effective in ischaemic stroke provided the treatment is started within the first three hours and in the absence of intracranial haemorrhage. ${ }^{8}$ SPECT may contribute to the treatment of the patient by confirming the ischaemia, indicating patients with a profound perfusion deficit who are at high risk of haemorrhagic transformation ${ }^{9}$ and demonstrating when thrombolysis is unnecessary because spontaneous reperfusion has already occurred (as in this patient). It is important to exclude cervical carotid or vertebral artery dissection because in this condition current recommended treatment entails early anticoagulation (despite the lack of formal trials) in an attempt to prevent further neurological deficit. ${ }^{10}$

The incidence of carotid artery damage in the form of dissection, thrombosis, and embolisation could be reduced in sport by increasing awareness of the vessel's vulnerability. Advice and supervision to avoid prolonged or vigorous neck holds are important. In conclusion this report has highlighted an important danger of stroke from neck trauma in the martial arts and emphasised some of the associated clinical features as well as current management issues.

Dr M O McCarron is supported by a Patrick Berthoud Fellowship.

1 Thomas P, Lowitt NR. A traumatic experience. $N$ Engl $\mathfrak{F}$ Med 1995;333:307-10.

2 Li MS, Smith BM, Espinosa J, Brown RA, Richardson P, Ford R. Nonpenetrating trauma to the carotid artery: seven cases and a literature review. $\mathcal{F}$ Trauma 1994;36:265-72.

3 Cantu RC. Head injuries in sport. Br $\mathcal{F}$ Sports Med 1996;30 289-96.

4 McLatchie GR, Davies JE, Caulley JH. Injuries in karate: a case for medical control. $\mathcal{F}$ Trauma 1980;20:956-8.

5 Mourad J, Girerd X, Safar M. Carotid-artery dissection after a prolonged telephone call. $N$ Engl f Med 1997;336: 516.

6 Alexandrov AV, Black SE, Ehrlich LE, Bladin CF, Smurawska LT, Pirisi A, et al. Simple visual analysis of brain perfusion on HMPAO SPECT predicts early outcome in acute stroke. Stroke 1996;27:1537-42.

7 Shimosegawa E, Hatazawa J, Inugami A. Cerebral infarction within six hours of onset: prediction of completed infarction with technetium-99m-HMPAO SPECT, $\not{J}$ Nuc Med 1994;35:1097-103.

8 The National Institute of Neurological Disorders and Stroke rt-PA Stroke Study Group. Tissue plasminogen activator for acute ischaemic stroke. $N \mathrm{Engl} f \mathrm{Med}$ 1995;333:1581-7.

9 Ueda T, Hatakeyama T, Kumon Y, Sakaki S, Uraoka T. Evaluation of risk of haemorrhage transformation in local intra-arterial thrombolysis in acute ischaemic stroke by initial SPECT. Stroke 1994;25:298-303.

10 Blunt SB, Galton C. Cervical carotid or vertebral artery dissection-an underdiagnosed cause of stroke in the young. $B M \mp$ 1997;314:243.

report should help to do this. Awareness in the sport of the vulnerability of the brain to carotid occlusion clearly needs to be highlighted. The authors hope that in future cases computed tomography and SPECT might all be sorted out within a three hour time window before a decision on thrombolysis. This seems a little optimistic, particularly in a UK context. 